\title{
Culture methods and environmental cues of human pluripotent stem cells
}

\author{
Hea-Jo Yoon* and Woo Jung Ho \\ Stem Cell Research Center, Apgujeong Miracle Clinic, South Korea
}

\section{Culture methods}

\section{Feeder based culture}

Standard human pluripotent stem cells (hPSCs) cultures have supportive cells such as inactivated mouse embryonic fibroblast (MEF) feeder cells that aid cell growth, secrete several important growth factors into the medium, which help maintain pluripotency and prevent differentiation [1]. Feeder based cultures are suitable for routine maintenance of hPSCs colonies, and MEFs are the most frequently used feeder cells, particularly because they support the robust growth of all types of embryonic stem cells as colonies [2]. However, since MEFs have complex and undefined heterogeneity, a variety of human cell types such as human fibroblasts, tubal, foreskin, and bone marrowderived stromal cells can be used as feeder cells instead of MEF [2]. Feeder based cultures are suitable for routine hPSCs maintenance, genetic engineering and single cell cloning, but are not used for clinical applications.

\section{Feeder-free culture}

Feeder-free culture, such as KSR supplemented with Activin A and FGF-2, has been developed which has been shown to support selfrenewal in the undifferentiated state of human embryonic stem cells [1].

\section{Non-colony type monolayer (NCM) expansion}

Single cell-based non-colony type monolayer (NCM) cultures have been developed since colony culture or suspension culture is not suitable due to disadvantages such as heterogeneity and suspension environment for drug screening or single cell analysis [3]. 2D monolayer cultures of pluripotent stem cells require sufficient cell numbers and cell density. HPSCs can be propagated by adhering monolayer to a weakly minus charged plastic container. Extracellular matrix proteins and proteoglycans are first secreted from the cells to form a matrix, which is attached to plastic containers such as negatively charged polystyrene. Cells are attached to the matrix through specific cell surface receptors on the cell surface. Thus, using existing culture vessels with better surfaces for attachment results in faster cell attachment [2].

Dissociated cells (single cells) at high density (ie, $\sim 1.4 \times 10^{5} / \mathrm{cm}$ $\left.{ }^{2}\right)$ were cultured on Matrigel coated polystyrene plates using Rho kinase (ROCK) inhibitor or JAK inhibitor 1, resulting in high single cell plating efficiency for early $24 \mathrm{~h}$. In addition, hPSCs can be cultured on NCM at the surface of human recombinant laminin-512 coated polystyrene without the use of a ROCK inhibitor, and generally hPSCs under these growth conditions have genetic stability and pluripotency [3].
The advantages of this culture are the feeder-free, adjustable growth rate, the production of homogeneous hPSCs, rapid cell growth, rapid (2-4 days) cell recovery after cryopreservation (vs. 1 to 3 weeks of colony culture). In particular, hPSCs grown with NCM are highly effective in teratoma formation and can be converted into colony-type culture if grown as clumps [2].

\section{Batch culture}

Batch incubation is a partially closed system in which most of the required material is loaded into the bioreactor vessel and only the substances necessary for gas exchange and $\mathrm{pH}$ adjustment are added or removed [2].

\section{Perfusion culture}

The perfusion culture is a culture method in which a new culture medium is continuously supplied by perfusion and the consumed medium is removed to thereby form a constant culture environment and enable cell culture for a much longer period of time [4].

\section{Suspension Cultures}

To expect the clinical effects of hPSCs, ranging from $10^{7}-10^{10}$ or beyond are essential. Suspension cultures of bioreactors enable large scale production of hPSCs for this purpose, and hPSCs grown by suspension culture remain pluripotent and chromosomally stable. Among many types of bioreactors, the spinner vessel and stirred-tank bioreactor provide a glass container with a working volume of $50 \mathrm{~mL}$ to $200 \mathrm{~L}$ and a homogeneous growth environment and can precisely control culture conditions by real-time monitoring of a temperature, oxygen level, acidity, growth factor consumptions, and metabolite concentrations [5]. A major disadvantage of suspension culture is cell damage associated with hydrodynamic shear forces [5]. Another disadvantage is that growth rates vary according to different suspension culture conditions. As the size and irregularity of cell aggregates increases, apoptotic cell loss, cell differentiation and heterogeneity may occur. In addition, the long-term effect of ROCK inhibition on hPSCs is unclear.

\section{Environmental cues}

In addition to the aforementioned culture media and extracellular matrix, there are physical and physiological environments that promote

*Correspondence to: Hea-Jo Yoon, MD, Stem Cell Research Center, Apgujeong Miracle Clinic, Seoul, South Korea, E-mail: heajo7890@daum.net

Received: January 25, 2019; Accepted: February 11, 2019; Published: February 13, 2019 
hPSCs growth such as temperature, humidity, osmosity, acidity, stiffness of growth surface, and cell density. Traditional hESC cultures proceed at normal oxygen levels whereas early in vivo mammalian embryos develop in hypoxic conditions. Low oxygen concentration $(2-3 \%)$ at the physiological level reduces spontaneous differentiation of hESCs, improves hESC clone recovery by up to 6 -fold and reduces chromosomal aberrations without affecting the expression of pluripotent markers [6]. Pluripotent stem cells have traditionally been grown on MEF on Matrigel as colonies and differentiate as embryoid body (EB) aggregates. The intercellular connections through intracellular signaling, cell density, ligand-receptor interaction, etc., have a great influence on the microenvironment of hPSCs. With the discovery of single cell death and survival mechanisms, various forms of culture can be selected, such as colonies, single-cell, single cell-based non-colony type monolayer, and suspended aggregates [1].

\section{References}

1. Chen KG, Mallon BS, McKay RD, Robey PG (2014) Human pluripotent stem cell culture: Considerations for maintenance, expansion, and therapeutics. Cell stem cell 14: 13-26. [Crossref]

2. Pamies D, Bal-Price A, Simeonov A, Tagle D, Allen D, et al. (2017) Good cell culture practice for stem cells and stem-cell-derived models. Alternatives to Animal Experimentation: ALTEX 34: 95-132. [Crossref]

3. Chen KG, Mallon BS, Hamilton RS, Kozhich OA, Park K, et al. (2012) Non-colony type monolayer culture of human embryonic stem cells. Stem cell research 9: 237-248 [Crossref]

4. Whitesides GM (2006) The origins and the future of microfluidics. Nature 442: 368 .

5. Serra M, Brito C, Correia C, Alves PM (2012) Process engineering of human pluripotent stem cells for clinical application. Trends in biotechnology 30: 350-359. [Crossref]

6. Forsyth NR, Musio A, Vezzoni P, Simpson AHR, Noble BS (2006) Physiologic oxygen enhances human embryonic stem cell clonal recovery and reduces chromosomal abnormalities. Cloning and stem cells 8: 16-23. [Crossref]

Copyright: (C2019 Yoon H. This is an open-access article distributed under the terms of the Creative Commons Attribution License, which permits unrestricted use, distribution, and reproduction in any medium, provided the original author and source are credited. 